\title{
Identification of novel aspartic proteases from Strongyloides ratti and characterisation of their evolutionary relationships, stage-specific expression and molecular structure Luciane V Mello* $^{* 1}$, Helen O'Meara ${ }^{\dagger 1,2}$, Daniel J Rigden ${ }^{1}$ and Steve Paterson ${ }^{1}$
}

\author{
Address: ${ }^{1}$ School of Biological Sciences, University of Liverpool, Crown Street, Liverpool L69 7ZB, UK and ${ }^{2}$ Department of Pharmacology and \\ Therapeutics, University of Liverpool, Ashton Street, Liverpool, L69 3GE, UK \\ Email: Luciane V Mello* - lumello@liverpool.ac.uk; Helen O'Meara - hevan@liv.ac.uk; Daniel J Rigden - drigden@liverpool.ac.uk; \\ Steve Paterson - S.Paterson@liverpool.ac.uk \\ * Corresponding author †Equal contributors
}

Published: 16 December 2009

BMC Genomics 2009, 10:611 doi:10.1186/1471-2164-10-611
Received: 27 April 2009

Accepted: 16 December 2009

This article is available from: http://www.biomedcentral.com/I47I-2/64/I0/6II

(C) 2009 Mello et al; licensee BioMed Central Ltd.

This is an Open Access article distributed under the terms of the Creative Commons Attribution License (http://creativecommons.org/licenses/by/2.0), which permits unrestricted use, distribution, and reproduction in any medium, provided the original work is properly cited.

\begin{abstract}
Background: Aspartic proteases are known to play an important role in the biology of nematode parasitism. This role is best characterised in blood-feeding nematodes, where they digest haemoglobin, but they are also likely to play important roles in the biology of nematode parasites that do not feed on blood. In the present work, we investigate the evolution and expression of aspartic proteases in Strongyloides ratti, which permits a unique comparison between parasitic and free-living adult forms within its life-cycle.
\end{abstract}

Results: We identified eight transcribed aspartic protease sequences and a further two genomic sequences and compared these to homologues in Caenorhabditis elegans and other nematode species. Phylogenetic analysis demonstrated a complex pattern of gene evolution, such that some $S$. ratti sequences had a one-to-one correspondence with orthologues of $C$. elegans but that lineagespecific expansions have occurred for other aspartic proteases in these two nematodes. These gene duplication events may have contributed to the adaptation of the two species to their different lifestyles. Among the set of $S$. ratti aspartic proteases were two closely-related isoforms that showed differential expression during different life stages: ASP-2A is highly expressed in parasitic females while ASP-2B is predominantly found in free-living adults. Molecular modelling of the ASP2 isoforms reveals that their substrate specificities are likely to be very similar, but that ASP-2B is more electrostatically negative over its entire molecular surface than ASP-2A. This characteristic may be related to different $\mathrm{pH}$ values of the environments in which these two isoforms operate.

Conclusions: We have demonstrated that $S$. ratti provides a powerful model to explore the genetic adaptations associated with parasitic versus free-living life-styles. We have discovered gene duplication of aspartic protease genes in Strongyloides and identified a pair of paralogues differentially expressed in either the parasitic or the free-living phase of the nematode life-cycle, consistent with an adaptive role for aspartic proteases in the evolution of nematode parasitism. 


\section{Background}

Adaptation of nematode taxa to a parasitic life-style represents an evolutionary challenge that is likely to be met, in part, by gene duplication in a gene family and subsequent acquisition of novel gene function among its paralogous members. One group of genes that has received considerable interest in this respect are aspartic proteases. Aspartic proteases are defined by having catalytic aspartic acid residues located in their active site clefts and include pepsins, renins, cathepsins $\mathrm{D}$ and $\mathrm{E}$ and chymosins [1-3]. In parasitic nematodes, aspartic proteases have been associated with digestion of host haemoglobin in the trichostrongylid Haemonchus contortus and in the hookworms Ancylostoma caninum and Necator americanus [4]. Moreover, the aspartic proteases of $A$. caninum and $N$. americanus each exhibit specificity in their ability to digest haemoglobin from natural versus unnatural hosts, indicating evolutionary adaptation to their host species [4]. Consequently, several aspartic proteases are currently being developed as vaccines in trichostrongylids and hookworms, many of which demonstrate an ability to reduce worm burden and/or egg output $[5,6]$. Aspartic proteases have also been shown to degrade skin macromolecules and aid skin penetration in hookworms, suggesting that their role in nematode parasitism is not limited to digestion of haemoglobin [7]. Given this, the aspartic proteases may play a role in the biology of nematode parasites that do not feed on blood, such as Strongyloides stercoralis, Onchocerca volvulus and Brugia malayi in which aspartic proteases have previously been identified $[7,8]$.

Nematodes of the genus Strongyloides infect a wide range of mammalian species, including humans and livestock, and infection occurs by skin penetration. Strongyloides spp. is not blood-feeding and parasitism is believed to have arisen separately in the lineages leading to Strongyloides and to the hookworms and trichostrongylids [9]. Strongyloides provides a useful model to study the evolution of genes associated with parasitism, since both parasitic and free-living adults are present in the life-cycle (Figure 1). Thus, one might predict that genes, including aspartic proteases, which retain distinct functions in freeliving and parasitic environments, are maintained within the Strongyloides genome. Previously, and in support of this prediction, we have presented preliminary evidence that distinct aspartic proteases from $S$. ratti, a laboratory model of Strongyloides infection, were differentially transcribed between free-living females and parasitic females [10].

In the present study we have identified ten aspartic proteases in $S$. ratti using sequence data from a collection of 14,761 cDNA clones described in Thompson et al. [11] and Evans et al. [10], by novel pyrosequencing of normalised cDNA using a 454-FLX sequencer and from an early

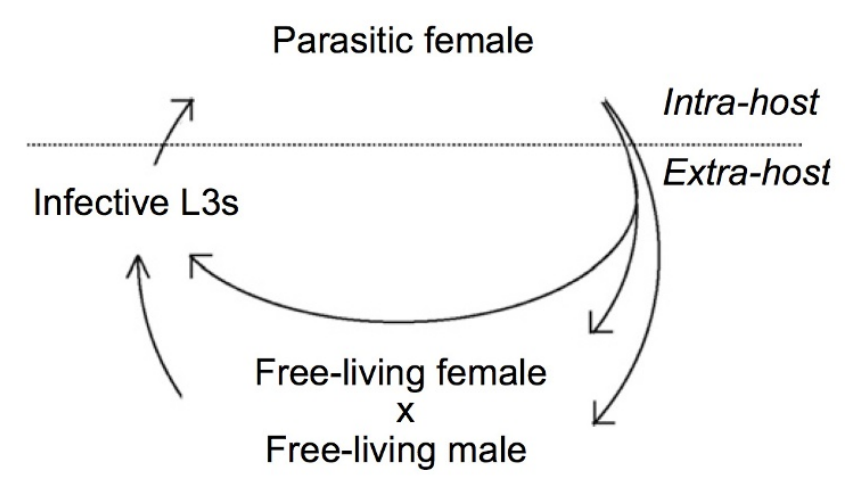

Figure I

Life-cycle of S. ratti. Eggs produced by mitotic parthenogenesis by parasitic females can develop either directly into iL3s by homogonic development or into free-living males and females, which reproduce sexually to produce eggs that develop into iL3s.

draft genome sequence of $S$. ratti http:/ www.sanger.ac.uk/sequencing/Strongyloides/. We used comparative sequence analysis and phylogenetic reconstruction between these and other aspartic proteases, including those belonging to the non-parasitic nematodes Caenorhabditis elegans and C. briggsae, to identify separate duplication events within different nematode lineages. We identified a pair of paralogous aspartic proteases in $S$. ratti (which we term ASP-2A and ASP-2B) that have arisen as a result of a recent duplication event but for which ASP$2 \mathrm{~A}$ is highly expressed in parasitic females while ASP-2B is highly expressed in free-living females, and we present a molecular model that allows this pair of paralogous genes to be placed in a structural context.

\section{Results \\ Identification of aspartic protease sequences in S. ratti} BLASTx searches of 3,688 contigs from $S$. ratti EST sequences (cDNA library) and 29,137 contigs from the pyrosequencing against the Wormpep, UniprotKB/SwissProt and nr databases revealed hits against various aspartic protease proteins. Upon close inspection of the consensus sequence for each contig, eight transcripts of aspartic protease were identified, which we termed ASP-1, ASP-2A, ASP-2B, ASP-3, ASP-4, ASP-7, ASP-9, and ASP-10. Two further putative asparatic protease genes, as $p-8$ and asp-11, were identified within the draft genome sequence for $S$. ratti (i.e. predicted genes for which there was no support from transcript evidence). 5' RACE was used to complete full length coding sequences for ASP-2A and ASP-2B, while the full length for other transcripts were obtained using the genome sequences for $S$. ratti, when necessary. The only exceptions were ASP-3 and ASP-4 where the present state of the genome sequence did not allow for completion at the N-terminus. Both nucleotide and 
amino-acid differences were observed across the length of all 10 sequences, indicating these isoforms represented distinct, paralogous loci rather than splice-site variants. Orthology was determined by the phylogenetic analysis and nomenclature was assigned to each gene, where possible, based on its orthology with the published C. elegans aspartic proteases, ASP-1 to ASP-6 [12] and to uncharacterised C. elegans aspartic proteases. Due to the difficulty in assigning orthologous $S$. ratti transcripts to C. elegans ASP-5 and ASP-6 based on our phylogenetic analysis (Cluster III discussion below), numbers 5 and 6 were not used. The eight $S$. ratti aspartic protease genes with their predicted amino acid sequences were submitted to GenBank (GenBank:FI756439, GenBank:FJ756440, GenBank:FJ756441，GenBank:FJ756442，GenBank:FJ756443， GenBank:FI756444，GenBank:Fu756445, and GenBank:ㅍ756446). All 454 cDNA sequence data are available from NEBC Envgen catalogue http://nebc.nox.ac.uk/ data/envbase. A Muscle alignment of the predicted amino acid sequences for each $S$. ratti aspartic protease is shown in Figure 2a. Signal peptides of the pre-proenzymes were determined and labelled in the alignment. It is known that aspartic proteases undergo a post-translational modification whereby about 60 residues are cleaved from the $\mathrm{N}$-terminus of the proenzyme producing the mature protein and the predicted cleavage points of these proteins are also marked in the alignment (Figure 2a). N-glycosylation site, presupposed to be necessary for lysosomal targeting, are also labelled, and as for C. elegans, the site is absent in ASP-3 and ASP-4. Syntichaki et al. [13] have shown these two aspartic proteases are involved in neurodegeneration in C. elegans.

\section{Phylogenetic analysis of aspartic proteases}

Protein distance-based phylogenetic analysis of the eukaryotic aspartic protease amino acid sequences resulted in an unrooted tree with three clusters (Figure 3 ). The C. elegans sequences for aspartic proteases were used as reference for the cluster division and interpretation as follows; Cluster I contained both ASP-3 and ASP-4; Cluster II C. elegans ASP-1; and Cluster III contained ASP-2, ASP-5 and ASP- 6 sequences. The tree could also be interpreted as two major groups, one containing ASP-1, ASP-3, ASP-4, and the other ASP-2, ASP-5 and ASP-6. Two sequences did not group with any other; Q8 MY59 from B. malayi and A8WZ33, an uncharacterised transcript in $C$. briggsae. There are also three uncharacterised transcript sequences, two from C. briggsae (A8X733 and A8XQ82) and one from C. elegans (Q18020) which forms a small group. Interestingly, the tree topology can be correlated with genomic position: in C. elegans, the ASP-1, ASP-2, ASP-5 and ASP-6 are located on chromosome V, whilst ASP-3 and ASP-4 are on chromosome X [12]. In the cases of branches containing C. elegans ASP-3 and ASP-4, each species is represented by a single sequence. This clear evo- lutionary relationship allows for the naming of the corresponding $S$. ratti transcripts.

A similar pattern was observed for ASP-1, although this gene is not well represented in terms of nematode species. As expected, ASP-1 from S. ratii and S. stercoralis are very similar, as shown by the tree. Remarkably, in the group containing C. elegans ASP-1 and four other hypothetical C. elegans proteins, C. briggsae is represented by ASP-1 alone. This suggests that there have been a number of duplications in the ASP-1 lineage in C. elegans since its divergence from C. briggsae. This scenario is supported by the fact that three of the C. elegans hypothetical proteins are genomic neighbours (Q8 MYN5, Q8 MYN6 and Q8 MYN7).

A different scenario was observed for the ASP-2, ASP-5 and ASP- 6 C. elegans sequences, the latter two of which are contained within a monophyletic group composed exclusively of Caenorhabditis sequences. This branches with a group of six $S$. ratti sequences, also clearly sharing a recent common origin. In a further branch are located two $H$. contortus sequences [14] which also group together. This suggests that at least three independent duplications have occurred in this part of the protease family, one in the lineage containing C. elegans, another in the predecessor of $H$. contortus, and a distinct set of events in the branch leading to $S$. ratti. Similar results were also obtained using minimum evolution and maximum parsimony phylogenetic approaches (not shown). These results mean that the naming of $S$. ratti sequences by orthology with their C. elegans counterparts breaks down here. Note that for other species other than C. elegans, numbering of aspartic proteases does not appear to be related to evolutionary history, instead being based, for example, on the order in which genes were discovered.

\section{Analysis of the ASP-2A and ASP-2B protein sequences}

A pair of paralogous $S$. ratti sequences, which we termed ASP-2A and ASP-2B, exhibited 76\% amino-acid similarity to each other and shared a conspicuous characteristic with C. elegans ASP-2: the presence of a cysteine rich insertion (see Figure 2a) near the beginning of the sequence, which consists of 30-35 amino acids located between the two putative catalytic residues [15]. Although these S. ratti sequences do not form a monophyletic cluster with C. elegans ASP-2, we named them ASP-2A and ASP-2B in recognition of this shared cysteine rich insertion. Of those genes represented in our phylogenetic analysis, the Cysrich insertion was present in: Q88NE0 and Q18020 of $C$. elegans; A8XV46, A8X733 and A8XQ82 of C. briggsae; Q9N9H4 of $N$. americanus; Q70JE2, Q25037 and Q9U8G6 of $H$. contortus; and ASP-2A and ASP-2B of $S$. ratti. Interestingly, the Cys-rich insertion has a quite sporadic distribution: Q18020, A8X733 and A8XQ82 form the small isolated group mentioned above, while all the 


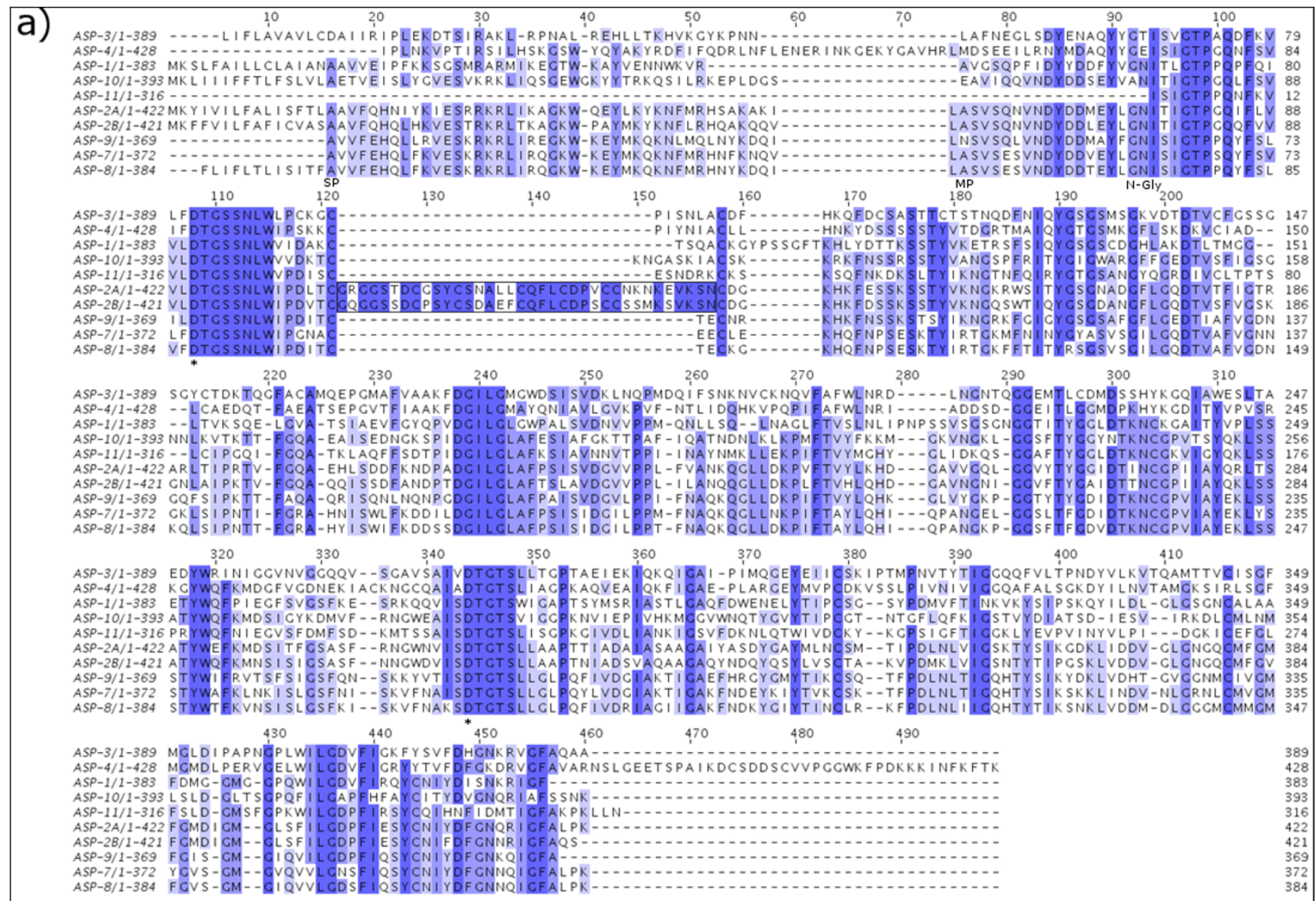

b)

\begin{tabular}{|c|c|}
\hline 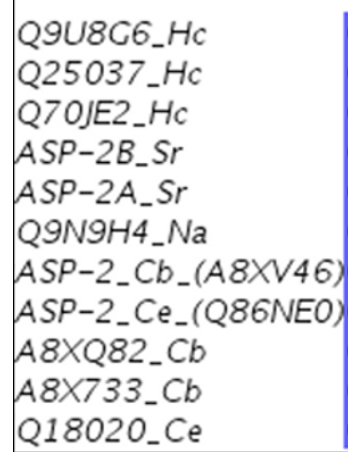 & 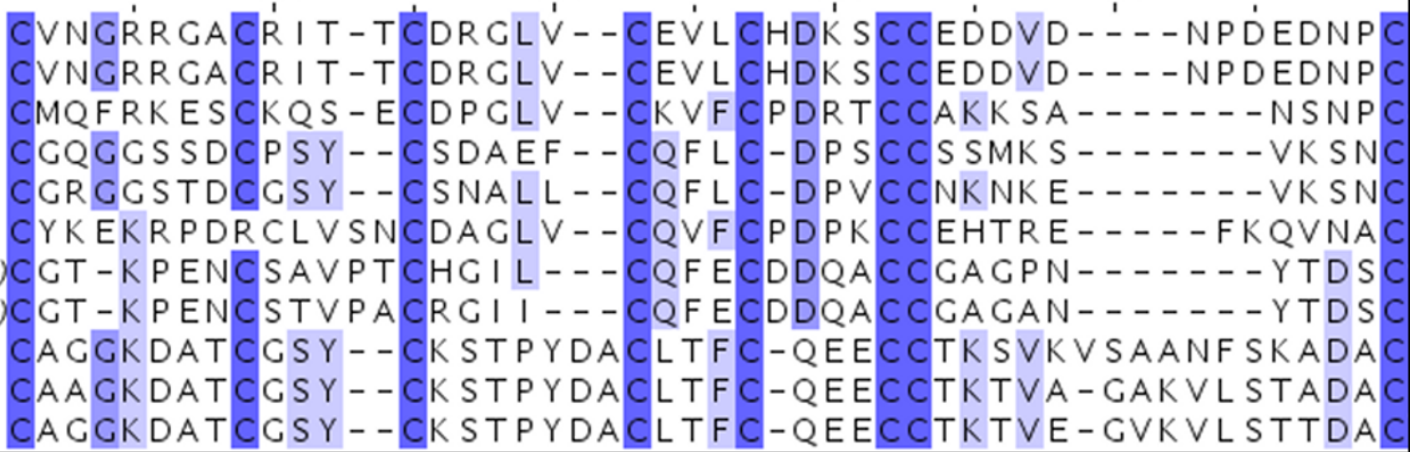 \\
\hline
\end{tabular}

Figure 2

Protein sequence alignment of aspartic proteases. (a) Derived protein sequences of the $10 \mathrm{~S}$. ratti aspartic protease aligned using MUSCLE [38]. Predicted signal peptide, the mature protein starting point, and conserved N-glycosylation sites are labelled SP, MP and N-Gly, respectively. The two catalytic aspartic acid residues are indicated by (*). Cys-rich region indicated by a box. (b) Alignment of the Cys-rich region from all species using MUSCLE [38]. Organisms are indicated after the accession numbers, as followed: $\mathrm{Cb}$, Caenorhabditis briggsae; Ce, Caenorhabditis elegans; Hc, Haemonchus contortus; Na; Necator americanus; $\mathrm{Sr}$, Strongyloides ratti. Figures were generated using JALVIEW [39]. 


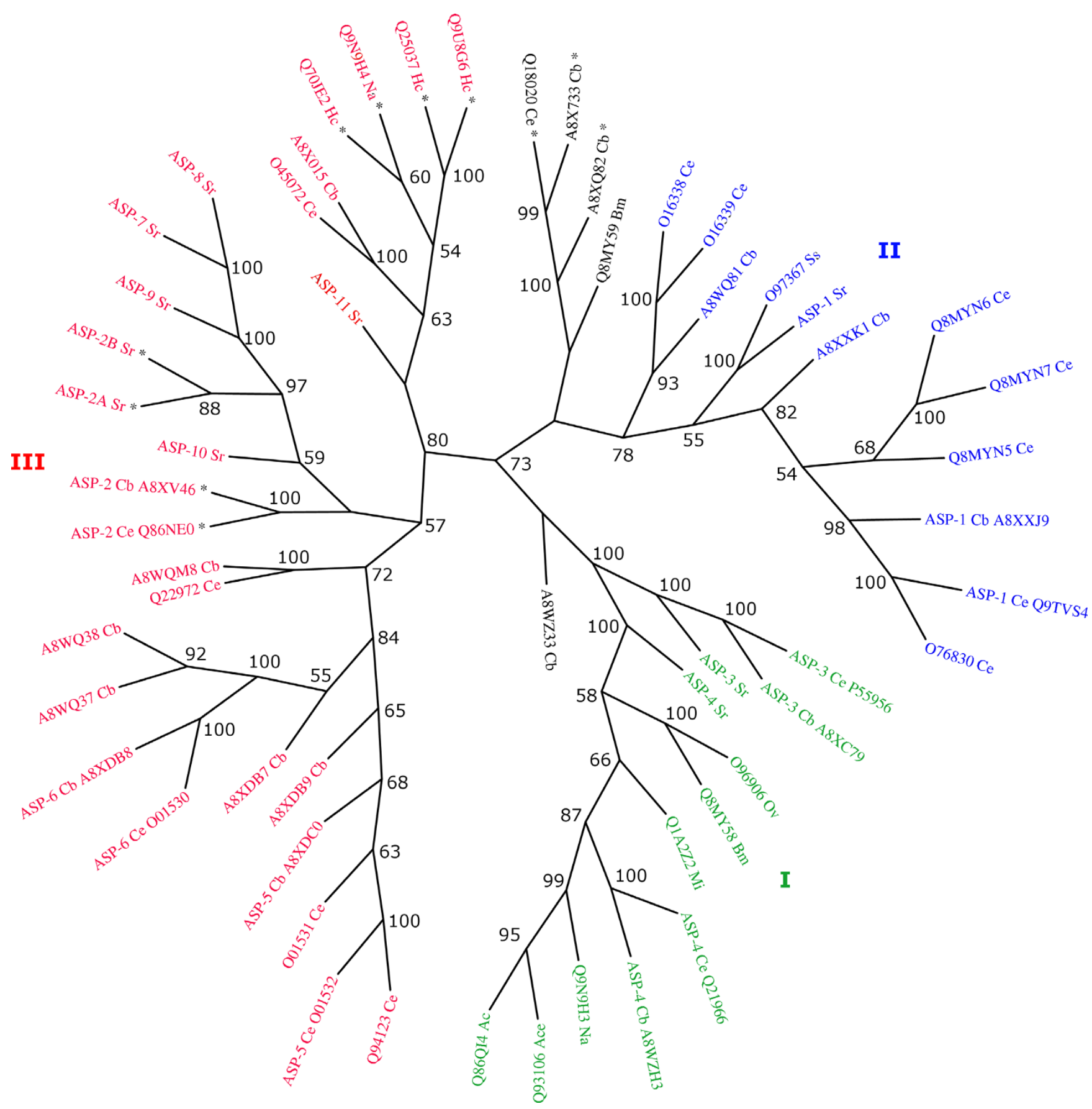

Figure 3

Unrooted phylogenetic tree. The eukaryotic aspartic protease domain, PF00026 was searched against the PFAM database [37] and sequences under the nematode branch were retrieved. Sequences were aligned using MUSCLE [38] and highly similar sequences were removed from the alignment. An unrooted neighbor-joining tree was calculated from protein distances using the MEGA package [40,4I]. Gapped regions were excluded in a complete fashion and percentage bootstrap values from 500 replicates were derived. Numbers above branches indicate the relative frequency in which bipartitions were observed (only values above $50 \%$ are shown). Sequences used in the final alignment are labelled according to their accession number at UNIPROT. Organisms are indicated after the accession numbers, as followed: Ac, Ancylostoma caninum; Ace, Ancylostoma ceylanicum; Bm, Brugia malayi; Cb, Caenorhabditis briggsae; Ce, Caenorhabditis elegans; Hc, Haemonchus contortus; Mi, Meloidogyne incognita; Na; Necator americanus; Ov, Onchocerca volvulus; Sr, Strongyloides ratti; Ss, Strongyloides stercoralis. Major groups I-III referred to in the text and coloured differently. All the sequences which present the Cys-rich insertion are labelled with a star. 
other sequences grouped within Cluster III of the phylogeny presented in Figure 3. Alignment of these sequences indicated that all Cys-rich insertions from these proteins shared a common ancestor: all contained 6 cysteine residues. With the exception of orthologous pairs from C. elegans and C. briggsae (Q86NE0 and A8XV46), a very recently duplicated pair from C. briggsae (A8XQ82 and A8X733) and the two H. contortus sequences (Q25037 and Q9U8G6), sequence identity between insertions was low at 21-39\% (Figure 2b). An alignment also indicated that Q88NE1 in C. elegans was identical to Q88NE0 except for the addition of $\sim 200$ residues at the $\mathrm{N}$-terminal (result not shown). However, it is unlikely that this longer protein is actually expressed. First, protein domain analysis reveal the presence of a partial eukaryotic aspartic protease domain coded for by the extra N-terminal residues, but structural analysis suggests that the N-terminal halfdomain would be unlikely to fold stably, since it lacks key components of the protein core. Also, a BLASTn search against the collection of $C$. elegans ESTs failed to reveal a single sequence spanning the junction between the additional N-terminal region and the complete aspartic protease domain common to Q88NE1 and Q86NE0. For $H$. contortus, Q9U8G6 and Q25037 share 98\% sequence identity and so are not likely to be true isoforms, but instead duplicated entries within UniProt. By contrast, $H$. contortus Q25037 and Q70JE2 share 50\% amino-acid sequence identity, and so are likely to represent distinct, paralogous loci.

\section{Analysis of differential transcription of aspartic proteases by $r$ PCR analysis}

Transcription of seven aspartic protease genes across the life cycle of $S$. ratti was measured by rtPCR (ASP-10 from the pyrosequencing analysis and aspartic proteases inferred by homology with the $S$. ratti genome, ASP-8 and ASP-11, were excluded). Figure 4 shows the relative expression of each gene between different life developmental stages. While transcription was observed for every gene in all stages, the expression of each transcript was variable between developmental stages. ASP-1 and ASP-3 were found to be the most constitutively transcribed in $S$. ratti. In C. elegans, ASP-1 expression is lower in adults than in larvae whereas C. elegans ASP-3 expression is relatively constitutive through development http://www.worm base.org. S. ratti ASP-4 transcription was markedly lower in the immature L1 and L2 stages than in iL3s or in any of the three adult stages, which is a similar pattern to that seen for C. elegans ASP-4 [16]. S. ratti ASP-7 and ASP-9 exhibited lower overall abundance than for the other $S$. ratti aspartic protease transcripts, and with levels somewhat elevated in the parasitic females (PAF) and the free

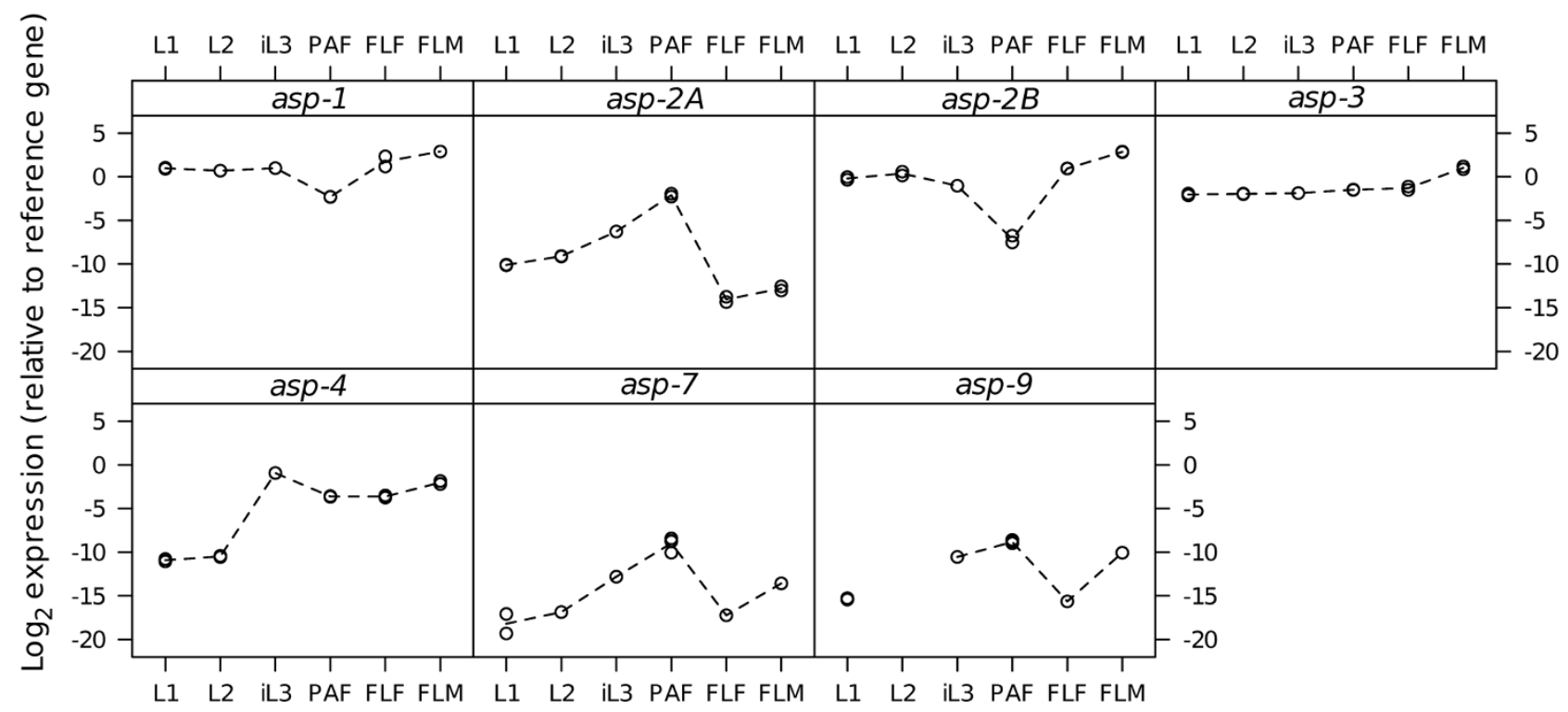

\section{Figure 4}

Transcription levels of the S. ratti aspartic proteases. The change in transcription levels of asp-I, asp-2A, asp-2B, asp-3, asp-4, asp-7 and asp-9 through different developmental stages measured by real-time PCR. Transcription levels for each stage are given as a $\log _{2}$ ratio relative to the reference gene (rpl20). The values of each biological replicate are plotted as a mean of of 3 technical replicates. 
living males (FLM). Pronounced differences in transcription levels between developmental stages were observed for the two S. ratti ASP-2 isoforms; with ASP-2A levels highest in the parasitic females (PAF) and lowest in the free living adults (FLF and FLM), whereas ASP-2B levels were lowest in PAF when compared with FLF and FLM. By contrast, C. elegans ASP-2 exhibits increasing expression from embryo through to adult [16].

\section{Molecular Modelling}

A model of the ASP-2A structure lacking the Cys-rich insertion was readily obtained, no doubt helped by the small size of the insertions and deletions in ASP-2A compared to the templates. Using a multi-template approach three insertions of 1,3 and 4 residues were modelled along with four small deletions of 1 or 2 residues (Figure 5 ). The model was stereochemically sound with no residues in Ramachandran-disallowed areas and $91 \%$ of residues in core regions of the Ramachandran plot. It had a DOPE score, an effective measure of protein structure quality in terms of residue contacts and solvent exposure, of -36610. This compares favourably with DOPE scores of -40670 and -39370 for the similarly sized main templates. Support for the accuracy of the alignment used comes from the observation that Cys residues 217 and 350, neither of which aligned with Cys residues in the templates, were found to be ideally positioned to form a novel disulphide bond (Figure 6).

The 30 residue Cys-rich insertion is far beyond the size limit of around 10-12 residues up to which reasonably accurate loop modelling is considered possible. Nevertheless, modelling was attempted using the predicted secondary structure (20 residues were predicted to adopt $\alpha$ helical conformation; Figure 5) and predicted disulphide bonding pattern as additional constraints. Unfortunately, there was no agreement between the results of the three disulphide bond predictors: each top prediction contained a different set of three predicted disulphide bonds. However, since the first three Cys residues of the insertion were predicted to lie within a single helix followed immediately by the fourth Cys residue, certain disulphides were rendered sterically impossible if the secondary structure prediction was assumed accurate. Models were therefore made using the insertion disulphide connectivities of (1$2),(3-4),(5-6)$, the top prediction of DIANNA [17] and $(1-2),(3-6),(4-5)$, the top scoring result from the Cysteine Separation Profile search algorithm at the PreCys server [18]. However, despite extensive efforts no model without obvious defects such as knots could be obtained so further analyses were applied to the model lacking this portion.

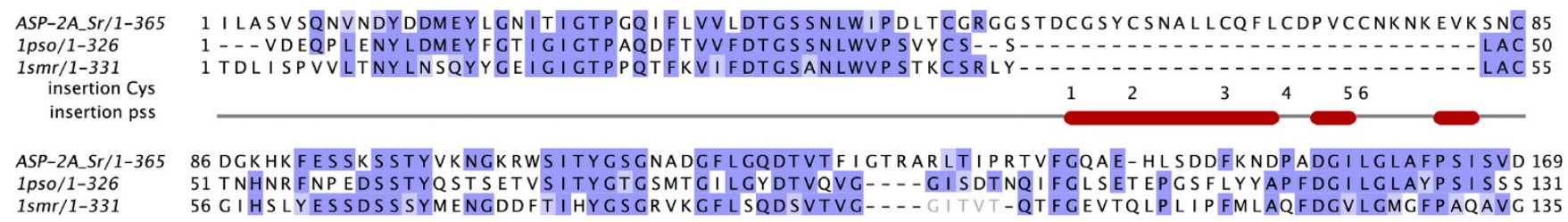

ASP-2A_Sr/1-365 170 GVVPPLFVANKQGLLDKPVFTVYLKHDGAVVGQLGGVYTYGGIDT I NCGP I I AYQRLTSATYWEFKMDS I TFCSASFR--NGWNV 252 1pso/1-326 132 GATPVFDN I WNOGLVSODLFSVYLSADD- - - S SG-SVVIFGG I DS SYYTGSLNWVPVTVEGYWO ITVDS I TMNGEA I ACAEGCOA 212 1smr/1-331 136 GVTPVFDHILSQGVLKEKVFSVYYNRGP - - HLLGGEVVLGGSDPQHYQGDFHYVSLSKTDSWQITMKGVSVGSSTLLCEEGCEV 217

ASP-2A_Sr/1-365 253 I SDTGT SLLAAPTT I ADA I A SAAGA I Y A SDYGAYMLNC SM- - T I P DLNLVIGSKTYS I KGDKLIDDVGLGNGQCMFCMFGMDIGM 335 \begin{tabular}{lll}
1 pso/1-326 & 213 \\
\hline
\end{tabular} 1smr/1-331 218 VVDTCSSFISAPTSSLKLIMQALGAKEKRL-HEYVVSCSQVPTLPDISFNLCGRAYTLSSTDYVLQR---DKLCTVALHAMDIPP 298

ASP-2A_Sr/1-365 $336--$ GLSFI LGDP F I ESYCN I YDFGNQR I GFAKS-

1pso/1-326 294 ESCELWI LGDVFIRQYFTVFDRANNQVGLAPVA

1smr/1-331 299 PTGPVWVLGATFIRKFYTEFDRHNNR IGFALAR

Figure 5

Protein sequence alignment between S. ratti ASP-2A and the principal templates used in its modelling. Alignment of ASP-2A with the principal templates used in its modelling, human pepsin in complex with pepstatin (PDB code Ipso; [44]) and mouse renin bound to the decapeptide inhibitor $\mathrm{CH}-66$ (Ismr; [45]). Shading indicates the degree of sequence similarity at each position. For the Cys-rich region, Cys residues are numbered and secondary structure predicted (using PSIPRED [53]; red indicates $\alpha$-helices) on lines below the alignment. Where only a single one of these two templates was used, the excluded template region is shown in light grey text. Boxed regions of ASP-2A indicate portions modelled using additional templates, Candida albicans aspartic proteinase 3 (PDB code 2h6t; [46]) for residues 247-249 and human renin (PDB code Ihrn; [47]) for residues 308-3II (see Materials and Methods). The figure was produced with Jalview [39]. 

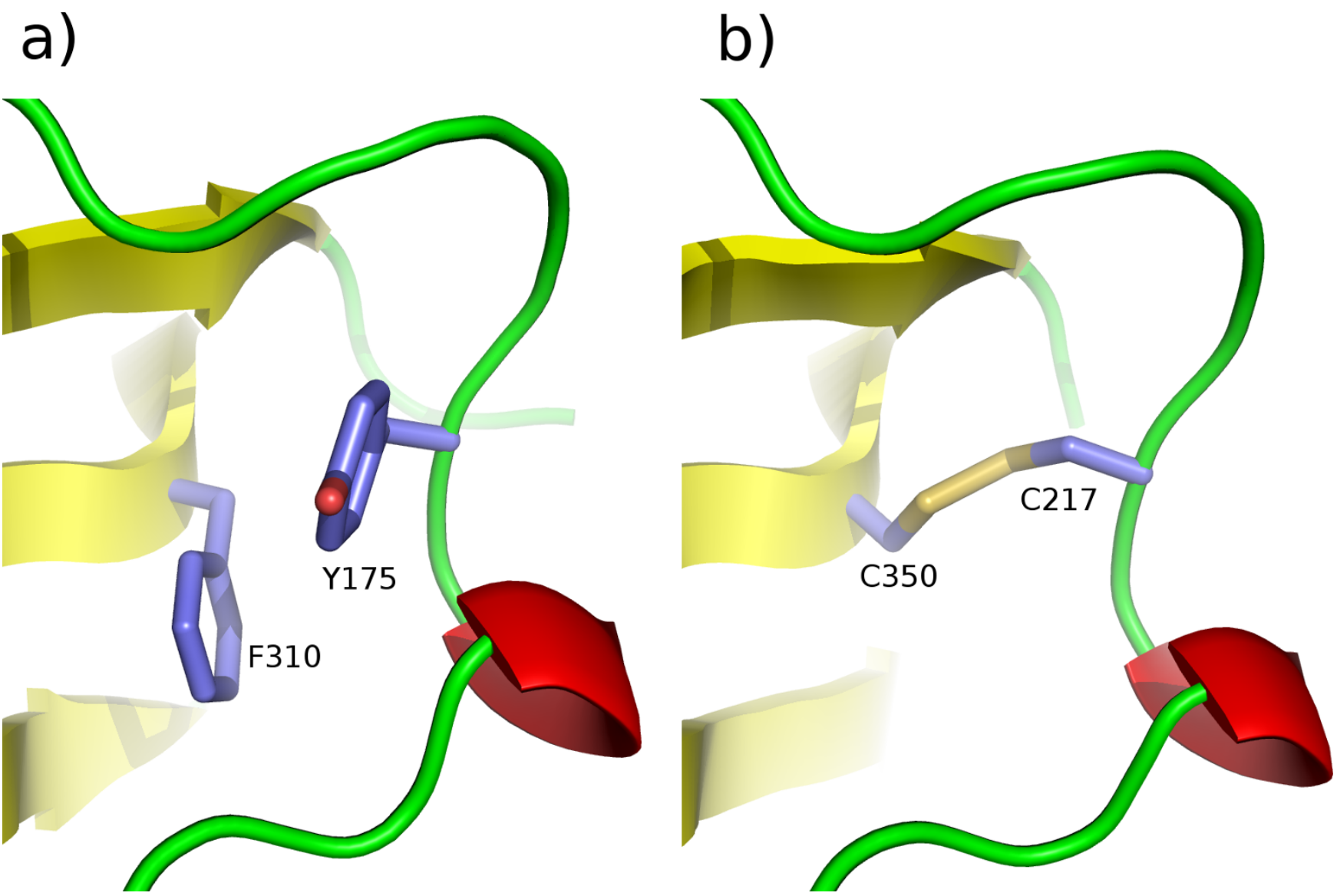

Figure 6

A putative novel disulphide bond in S. ratti ASP-2A. Comparison of human pepsin template (PDB code Ipso; [44]) and ASP-2A model in the vicinity of the ASP-2A-specific novel putative disulphide bond. The figure was produced using PyMOL [49], as were Figures 8 and 9.

Of the 335 modelled residues of ASP-2A, 74 differ in ASP$2 \mathrm{~B}$. Mapping of these on to the ASP-2 modelled structure (Figure 7) shows that they contain more solvent-exposed residues than buried ones so that the $3 \mathrm{D}$ structures of the two isoforms may be assumed to be very similar. The differing residues are well dispersed over the molecular surface but, importantly, only three positions are predicted to lie near to the substrate-binding cleft. These are Met15, Thr79 and Ile136 in 2A and are replaced by Ile, Gln and Leu respectively in ASP-2B. These are small to moderate differences in size and physicochemical characteristics, suggesting that substrate specificity will be very similar between the proteins

While analysing the differences between ASP-2A and ASP$2 \mathrm{~B}$, it was observed that among the 22 replacements resulting in charge differences there was a marked preference for positive residues in ASP-2A to be replaced by neutral ones in ASP-2B, or neutral positions in ASP-2A to match negatively-charged residues in ASP-2B. At these 22 positions ASP-2A contains 11 positively charged residues and six negatively charged ones, compared to the figures for ASP-2B of two and three, respectively. This results in a net charge difference on the protein as a whole of -6 in favour of ASP-2B. Electrostatic surface mapping of the ASP-2A model shows that it, in common with other aspartic proteases including both templates (not shown) is strongly negatively charged, on both faces of the molecule but in particular at the substrate binding cleft. Comparison with ASP-2B (Figure 8) shows that the latter's additional negative charge is spread over several parts of the molecular surface. Although we were unable to model the Cys-rich insertion, sequence analysis shows that it follows a similar trend towards more negative charge on ASP-2B vs ASP-2A: of the three isoform sequence differences resulting in charge changes, two are from neutral (ASP$2 \mathrm{~A})$ to negative (ASP-2B) and the third from positive to neutral. 


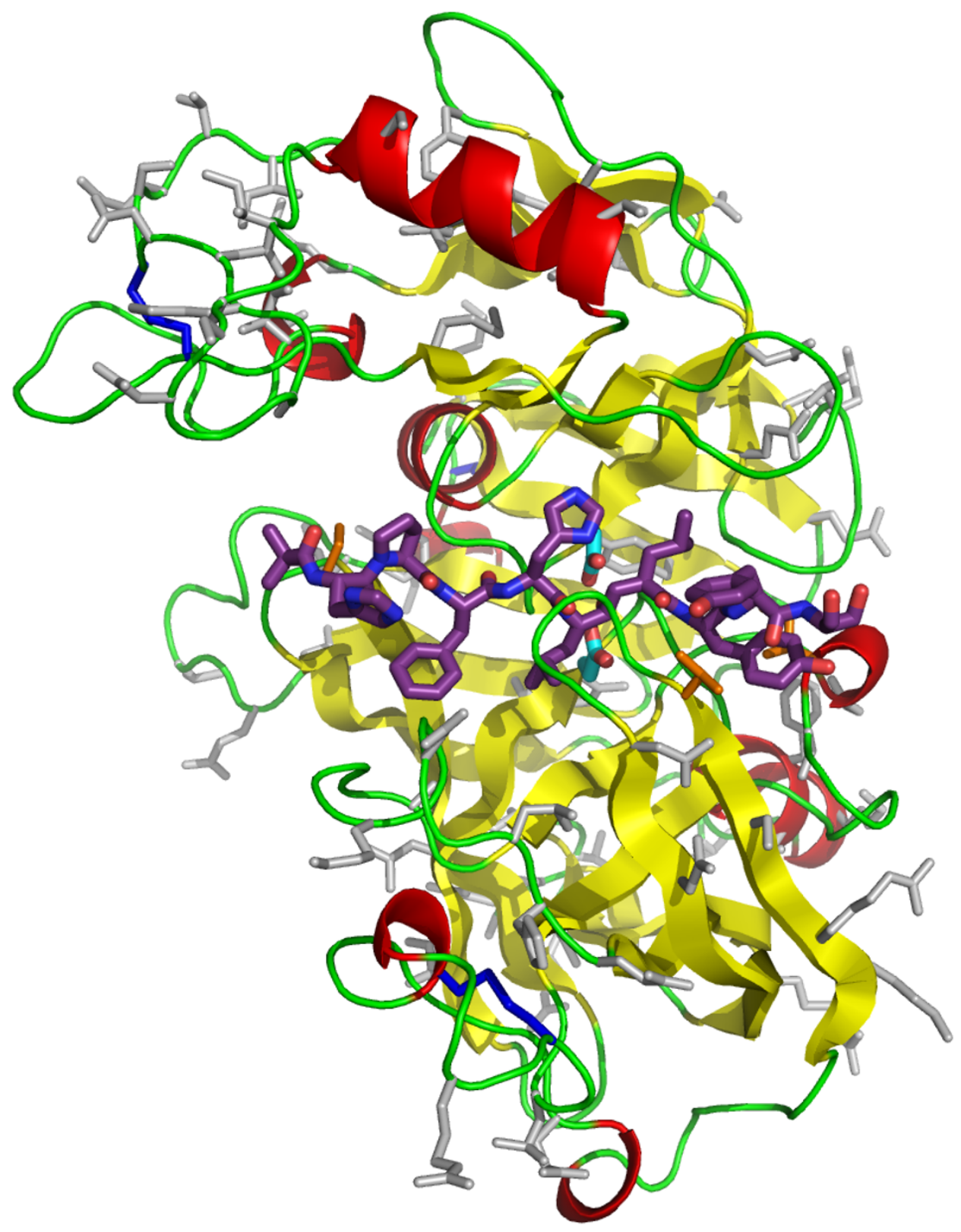

Figure 7

Cartoon view of the final ASP-2A model coloured according to secondary structure (yellow $\beta$-strand, red $\alpha$ helix and green irregular). For the purposes of orientation, the inhibitor from superimposed mouse renin template is shown as purple sticks. Catalytic aspartic acid residues are shown as cyan and red sticks and disulphide bonds as dark blue sticks. Side chains that differ in ASP_2B are shown as orange (near catalytic site) or grey (elsewhere) sticks.

\section{Discussion}

We found eight distinct asparatic protease genes transcribed by $S$. ratti. A key question is whether such diversity within the asparatic protease family has arisen recently, particularly within lineages leading to parasitic nematodes, or whether this diversity predated the evolution of the nematode phylum and has been preserved since. While based on a possibly incomplete sampling of $S$. ratti aspartic proteases and a possibly incomplete annotation of aspartic proteases in genome sequences, our phylogenetic analysis suggests three clusters of asparatic proteases. Two of these (Clusters I and II in Figure 3) represent anciently derived aspartic proteases and includes ASP-1, ASP-3 and ASP-4 of C. elegans, corresponding orthologs for all three of these genes from $S$. ratti and additional orthologous genes in C. briggsae, Brugia malayi, Onchocerca 

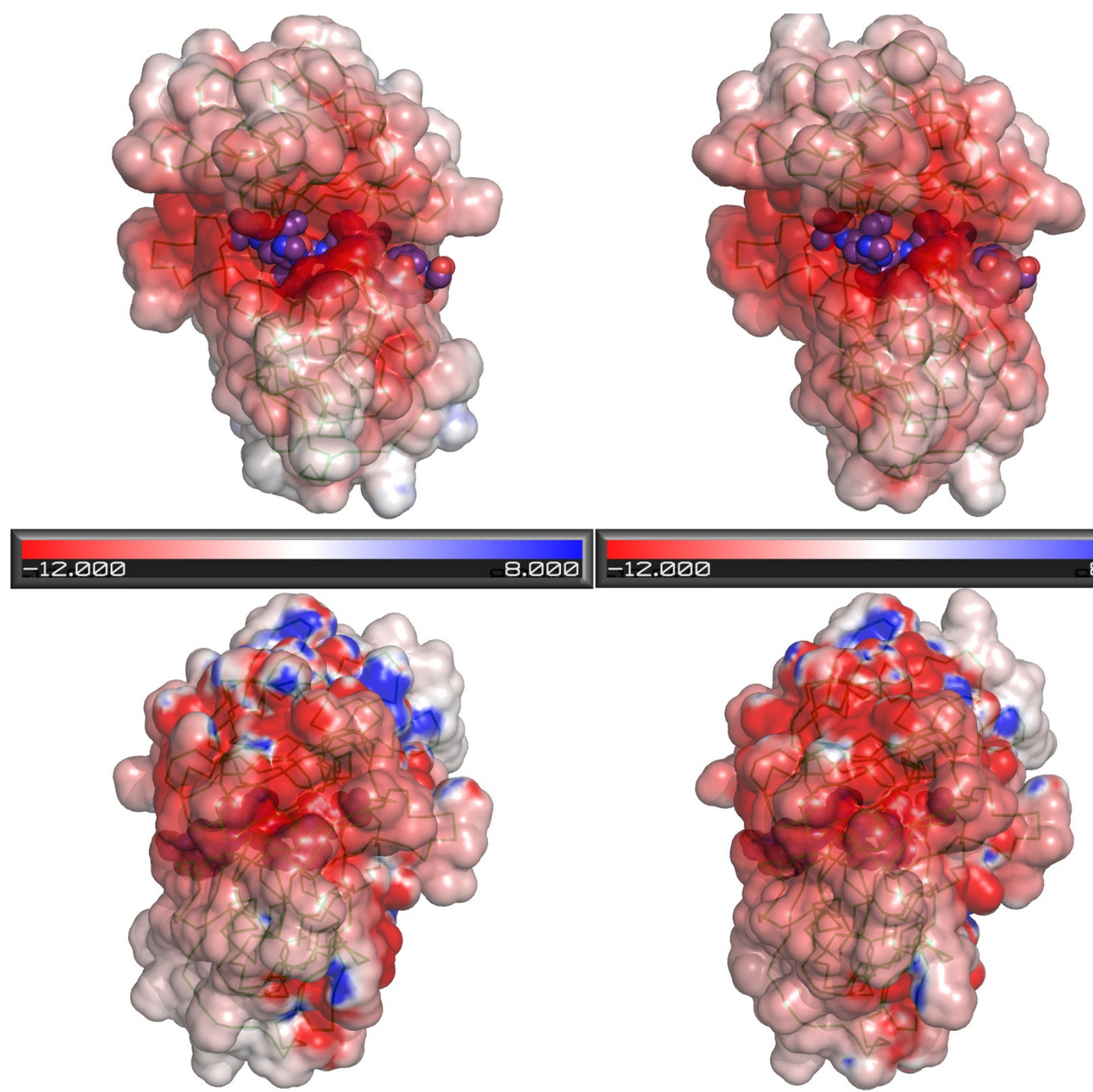

\section{Figure 8}

Comparison of electrostatic surfaces of models of ASP-2A (left) and ASP-2B (right). Upper panels show the side of the molecule containing the catalytic site: the inhibitor from superimposed mouse renin template is shown in ball and stick representation. Lower panels show the opposite face of the molecules. Red colouring indicates negative charge and blue positive charge. Electrostatic calculations were done with APBS [55].

volvulus, Necator americanus, Ancylostoma caninum, Meloidogyne incognita and $S$. stercoralis. The remaining five $S$. ratti transcripts form a monophyletic group within Cluster III (at least within those sequences available to the phylogenic analysis). The diversity among these five $S$. ratti tran- scripts has therefore arisen from a more recent set of duplication events than those present in Clusters I and II. Similarly within Cluster III, independent duplication events in C. elegans and C. briggsae can be inferred, while aspartic proteases from the strongylids $N$. americanus and 
H. contortus also group together. Thus, the evolution of aspartic proteases in Cluster III appears more dynamic than in Clusters I and II, involving the repeated duplication of asparatic proteases in different lineages.

Two of the S. ratti transcripts within Cluster III, ASP-2A and ASP-2B, appear to have arisen from a relatively recent duplication, share $76 \%$ amino acid similarity to each other and exhibit a markedly different expression pattern (Figure 4). Thus, ASP-2A is predominantly found in parasitic females and ASP-2B in free-living females, suggesting that these paralogs may have different functions in parasitic versus free-living females. We investigated the structure-function relationship of $S$. ratti ASP-2A and ASP-2B by homology modelling. Since only three of the ASP-2A/ ASP-2B sequence differences were located at the substrate binding cleft, and those were relatively conservative changes, it can be concluded that the substrate specificity of the isoforms is likely to be very similar. One possible explanation for the fixation of two copies of the gene with similar specificity may lie with the analysis of their electrostatic properties. Although both are strongly negatively charged at neutral $\mathrm{pH}$, sequence differences consistently led to extra negative change on isoform ASP-2B. It is possible that these electrostatic differences reflect differences in the $\mathrm{pH}$ at which the two isoforms operate. Most intestinal proteases from C. elegans exhibit optimal activity at $\mathrm{pH} 5$, suggesting that the C. elegans intestinal lumen is mildly acidic [19], which is consistent with the negatively charged surface of $S$. ratti ASP-2A and ASP-2B. Little is known about nematode digestion [20], but it is possible that the $\mathrm{pH}$ of the intestinal lumen of $S$. ratti parasitic adults is less acidic than that of free-living adults, perhaps due to different requirements between digestion of microbiota by free-living stages versus host-derived nutrients by parasitic stages. Accordingly, to maintain solubility and activity, the free living-specific isoform ASP-2B, might have adjusted its pI downwards. It also remains possible that the two isoforms differ in their interaction with as-yet unidentified elements of their different environments, since inter-isoform differences are found throughout the surface of the protein (Figure 7).

Notably, S. ratti ASP-2A and ASP-2B share a cysteine rich insertion with each other and with ASP-2 of C. elegans (Q86NE0), C. briggsae (A8XV46), Q9N9H4 of N. americanus and Q70JE2 and Q25037 of H. contortus. These aspartic proteases from $N$. americanus and $H$. contortus have previously been implicated in parasitism, particularly the digestion of haemoglobin $[4,14,21]$. Moreover, Q25037 of $H$. contortus is almost exclusively expressed by the blood-feeding stages (late L4 and adults) of Haemonchus contortus, and is localised in the lumen of the gut of the adult parasite [14]. Since $S$. ratti does not feed on blood, the role of ASP-2A in $S$. ratti parasitic females remains unclear, but may involve the digestion of other host macromolecules. The Cys-rich insertion, the similarity of which between enzymes illustrates a single evolutionary origin (Figure 2b), shows a rather sporadic distribution in proteases from Cluster III and is also present in the group of Q18020, A8X733 and A8XQ82 which do not reliably fall within any of the three main clusters. Importantly, different phylogenetic methods, including calculation of differences between sequences by both pair-wise and complete deletion methods, yield the same topology. The absence of the expected close relationship between proteases sharing this prominent sequence feature indicates that the insertion must have been present in the common ancestor of them all, but lost several times during evolution. The repeated loss of this prominent feature is itself concomitant with the repeated duplication of genes within Cluster III. This impression of ongoing genetic flux in the nematodes is in accord with previous analyses of other phenomena such as gene duplication and diversification [22], gene movement between chromosomes [23] and gene deletions and truncations [24].

Our results from $S$. ratti demonstrate that transcriptomic and genomic sequence data can be combined to identify distinct members of a gene family. Such data are increasingly becoming available for other parasitic nematodes, particularly in light of concerted efforts by major sequencing centres and with the advent of new pyrosequencing techniques [25]. There will therefore soon be the opportunity to perform comparative evolutionary analyses and to examine the processes of gene gain and loss acting on large numbers of gene families across multiple nematode species. Doing so will provide fresh insights into the genetic and evolutionary mechanisms associated with parasitism. However, such comparative evolutionary analyses will also bring considerable challenges. Although we have used a number of bioinformatic methods to identify and align aspartic protease sequences, these methods have required manual supervision to construct reliable phylogenies. A more automated approach, suitable for analysis of multiple gene families, will still require validation by a manually supervised approach. Our results also highlight the inherent difficulties in imposing a consistent nomenclature on duplicated members of gene families. Ideally, one would wish the nomenclature to reflect the evolutionary relationships between the orthologs, perhaps by associating parasitic nematode genes to their corresponding C. elegans ortholog. However, as is the case for $S$. ratti aspartic proteases in Cluster III, gene duplications in lineages leading to parasitic nematodes generate paralogs and so confound a nomenclature based on orthology to a reference sequence genome. Finally, the type of bioinformatic and evolutionary analyses that we present here can highlight genes, such as $S$. ratti ASP-2A, which may have acquired a significant role during the evolution of 
parasitism. As such, these analyses help guide and inform - but are not a substitute for - more detailed molecular and biochemical work to examine in greater depth the novel functions acquired by such duplicated genes that aid a parasitic life-style.

\section{Conclusions}

S. ratti provides a powerful model to explore the genetic adaptations associated with the evolution of nematode parasitism due to the presence of both parasitic and freeliving adults within its life-cycle. We have examined the role of gene duplication in adaptation to the parasitic lifestyle by analysis of genes from the aspartic protease family in Strongyloides and identified a pair of paralogues differentially expressed in either the parasitic or the free-living phase of the nematode life-cycle, consistent with an important role for aspartic proteases in the evolution of nematode parasitism. While our findings should be tempered by the possible incompleteness of some databases and their annotation, we consider that gene duplication, accompanied by the acquisition of new function, is likely to be more widely applicable to understanding the evolutionary adaptations and biology of nematode parasites.

\section{Methods}

\section{Collection of S. ratti material}

A laboratory line (LIV 4) of S. ratti was used for all analyses. This line was generated and maintained in female Wistar rats (Charles River, UK) as described previously $[26,27]$. For the collection of free living stages, faeces from 8 -day infected rats was collected and cultured at $19^{\circ} \mathrm{C}$ [26]. L1, L2 and iL3 stages were removed from the faeces on days 9, 10 and 11 post infection (p.i.) using the methods described by Paterson and Viney [28], whereas free living adult males and females were picked by pipette on day 12 p.i. For the collection of parasitic females, 8-day infected rats were starved overnight and then culled on day 9 p.i. Parasitic females were removed from the small intestines and counted as described by Evans et al. [10]. Worms from each stage were placed in $500 \mu \mathrm{l}$ TRIzol reagent (Invitrogen Life Technologies, Paisley, UK) and stored at $-80^{\circ} \mathrm{C}$. Total RNA was then extracted using TRIzol Plus extraction columns (Invitrogen Life Technologies, Paisley, UK) following the manufacturer's instructions. All animal work was conducted under United Kingdom Home Office licence (PPL40/2933) and in accordance with the University of Liverpool's ethical review procedures.

\section{Transcript sequence data from cDNA clones}

Transcript sequence data was used from a $S$. ratti cDNA library constructed from 14,761 cDNA clones selected from five stages during parasitic and free-living development as described in Thompson et al. [11]. Expressed sequence tags (ESTs), derived by Sanger sequencing, from these clones were assembled into 3,688 unique transcripts or 'contigs', as described by Evans et al. [10].

\section{Transcript sequence data from pyrosequencing}

$0.7 \mu \mathrm{g}$ total RNA extracted from adult free living male and female, and parasitic female worms was mixed to form 2.1 $\mu \mathrm{g}$ and then shipped in ethanol to Evrogen Technologies, Moscow. The sample was subjected to SMART cDNA synthesis and amplification [29], followed by Duplex-Specific-Nuclease (DSN) normalisation [30] in order to enrich for full length transcript sequences and equalise transcript abundance. Upon return, $80 \mathrm{ng}$ of normalised sample was subjected to a further round of amplification using the Advantage 2 Polymerase mix and $0.4 \mu \mathrm{M}$ SMART PCR primer (Clontech Laboratories, France) following the manufacturer's instructions. The resulting product was purified using QIAquick PCR purification columns (Qiagen, Crawley, UK) to a final concentration of $\sim 300 \mathrm{ng} / \mu \mathrm{l}$ prior to pyrosequencing sequencing. At least $4 \mu \mathrm{g}$ of normalised cDNA was submitted to the Advanced Genomics Facility (AGF) at The University of Liverpool where the sample was prepared using methods essentially as described by Margulies et al. [31]. Briefly, sample quality was first assessed using the Agilent bioanalyser and then the sample was nebulised into 300-500 bp fragments, blunted and ligated to short adapters. Each fragment was then fixed to DNA capture beads (one fragment one bead) and subjected to emulsion PCR (emPCR). All fragments were amplified in parallel and then placed into individual wells of a PicoTiterPlate where they were sequenced also in parallel using the Genome Sequencer FLX Instrument (Roche). Contig assembly was carried out using the Roche Newbler assembly programme, software version 1.1.03.24.

\section{Genome sequence data}

Data from an early draft of $S$. ratti genome sequence was used, generated by the Sanger Institute, UK. This consisted of 437,784 shotgun reads comprising $281 \mathrm{Mb}$ of total sequence assembled into 9,792 contigs with a combined length of $37 \mathrm{Mb}$ http://www.sanger.ac.uk/sequencing/ Strongyloides.

\section{Identification of aspartic protease transcripts within S. ratti transcript sequence data}

Similarity searches were conducted on the consensus sequence of each contig built for the cDNA library and for the results of pyrosequencing to assign putative identities. For this, BLASTx [32] searches with a cut-off of $e<1 \times 10^{-}$ 10, against wormpep196 http://ftp.sanger.ac.uk/pub/ wormbase[33], UniprotKB/Swiss-Prot http://www.uni prot.org[34] and $\mathrm{nr}$ http://www.ncbi.nlm.nih.gov[35] were carried out. The databases used were those available during September 2008. Where incomplete protein sequences were obtained, possible erroneous frame shifts 
were sought using the translate tool at the Expasy website http://www.expasy.org/tools/dna.html and, where found, manually corrected. Only transcripts that could be unambiguously generated in this way were considered for further analysis. In some cases, the $5^{\prime}$ end of transcripts were missing and here a BLASTn search with a cut-off of $e<1 \times$ $10^{-10}$ was carried out against the draft $S$. ratti genome.

\section{Identification of aspartic proteases within S. ratti genome sequence data}

The protein sequence of the ASP-1 for C. elegans was used to search against the nr database using a cut-off of $e<1 \times$ $10^{-10}$. A PSI-BLAST [32] profile was constructed over 4 iterations. This protein profile was then used to search against the $S$. ratti genome draft by PSI-tBLASTn. The gene finder program FGENESH [36] was used to look for possible aspartic proteases in the genome. These approaches were used in order to obtain any genomic sequence which may encode for aspartic protease but not sampled by the cDNA library or by the pyrosequencing method.

\section{Phylogenetic analyses}

The Pfam database http://pfam.sanger.ac.uk/[37] was searched for the eukaryotic aspartic protease domain, PF00026, and all sequences listed under nematode and human branch of the tree were retrieved. Partial sequences were once again removed, as well as redundant sequences and those representing the human cathepsins E (not relevant for the present work). In addition, a rigorous inspection to remove duplicated entries in the database was also performed. However, some entries were retained where it was unclear whether they represented duplicated entries or duplicated genes. Thus, for C. elegans and C. briggsae aspartic proteases, the 6 well characterised aspartic proteases plus 12 (C. elegans) and 11 (C. briggsae) putative proteins inferred from cDNA sequences were included in the sequence analysis. We did not include C. briggsae entry A8XDC1 which is clearly incomplete. Sequences A8XDB7, A8XDC0 (ASP-5_ Cb) and A8WZH3 (ASP-4_Cb), were included in the phylogenetic analysis although we note there are some doubts regarding the accuracy of these sequences: for example, the sequence of A8XDC0 may contain a frame-shift towards the C-terminus. For H. contortus, two entries which has high identity between them (Q25037 and Q9U8G6) were included in the sequence analysis. Amino acid alignments were produced from a total of 68 sequences, and 56 (excluding the human sequences), including eight from $S$. ratti with transcript evidence and 2 inferred by homology in the $S$. ratti genome. Alignments were built using the MUSCLE program [38] and manipulated using the JALVIEW program [39]. Phylogenetic analyses were performed by calculation of a protein distance matrix followed by application of the neighbor-joining method using the MEGA package $[40,41]$ : gapped regions were excluded in a complete fash- ion and percentage bootstrap values from 500 replicates were derived.

\section{5 ' sequencing by Rapid Amplification of CDNA ends (RACE-PCR)}

Using the information given in the contig sequences, gene specific primers were designed to amplify the 5 ' ends of $a s p-2 A$ and $a s p-2 B$ by RACE-PCR (Additional File 1 ). $1^{\text {st }}$ strand cDNA was synthesised as described for pyrosequencing. For second strand synthesis, $0.2 \mu \mathrm{M}$ SMART PCR primer (Clontech Laboratories, France) was used with $0.4 \mu \mathrm{M}$ gene specific primer to amplify a $200 \mathrm{bp}$ product for both genes. Each product was purified using QIAquick PCR purification columns (Qiagen, Crawley, UK) and $100 \mathrm{ng}$ sent to MWG-Biotech, Germany for sequencing.

\section{Analysis of transcription abundance by realtime $P C R$ (rtPCR) analysis}

$1^{\text {st }}$ strand CDNA was produced using total RNA from the different $S$. ratti life stages following protocols previously described [10]. Oligonucleotide primer pairs specific for each aspartic protease gene were designed (Additional File 1) using the Primer Express ${ }^{\mathrm{TM}}$ program (Applied Biosystems, Warrington, UK) to amplify a 60-90 bp insert with an annealing temperature of $60^{\circ} \mathrm{C}$. Primers specific for the small ribosomal subunit S20 ( $\mathrm{rpl} 20)$ gene were also designed and used as a reference for normalisation of the data [10]. rtPCR analysis was performed on all samples using protocols also previously described [10] and for each life stage the relative abundance of each transcript was calculated. Three technical replicates were performed for each gene on all samples. Biological replication was available for the L1, L2, parasitic females, free living females and free living males (two replicates each) but not for the iL3s.

\section{Molecular Modelling}

The profile-profile matching server HHPRED [42] was used to select the most favourable templates for model building of ASP-2A from the many available aspartic protease structures. It showed that pepsins were the most closely related available structures followed by renins. Complexed structures, of human pepsin in complex with pepstatin (Protein Data Bank [PDB; [43]] code 1pso; [44]) and of mouse renin bound to the decapeptide inhibitor CH-66 (PDB code $1 \mathrm{smr} ;$ [45]) were chosen as the principal templates. The pepsin was $38 \%$ sequence identical to ASP-2A, while for renin the figure was 34\%. For the bulk of the ASP-2A model structure both templates were simultaneously used, but they differed in the size of several loop regions. In those places a single template was used based on local sequence similarity with ASP-2A. For another loop a lower-ranked structure, Candida albicans aspartic proteinase 3 (PDB code $2 \mathrm{~h} 6 \mathrm{t}$; [46]) was used to provide a 
loop of the same length as ASP-2A. For a further region the structure of human renin (PDB code 1 hrn; [47]) was used to provide a template where a portion of the mouse renin structure had not been localised in electron density maps. The positions of insertions and deletions in ASP-2A with respect to the templates were inspected and, where necessary, suitably manually repositioned. Templates were superimposed using SSM [48] and inspected using PyMOL [49]. Manual adjustments to the HHPREDderived sequence alignments of ASP-2A with templates were made using JALVIEW [39].

Modelling was carried out with MODELLER 9v5 [50] initially for the ASP-2A sequence lacking the Cys-rich insertion. In the first models it became apparent that Cys217 and Cys350, neither aligning with Cys residues in any of the templates, were suitably positioned to form a disulphide bridge. This bond was manually specified in a second modelling round during which ten models were constructed. These derived from a $3 \AA$ coordinate randomisation procedure applied to the initial crude model generated by MODELLER. This is an established method for exploring conformational space. The stereochemical quality of the models was assessed with PROCHECK [51] and the single model without any residues in disallowed regions of the Ramachandran plot was selected. Constraints on the structure of the Cys-rich insertion that could help modelling were sought from two sources - predictions of disulphide bonding patterns (based on the reasonable assumption that the Cys residues in the insertion form intramolecular disulphide bonds in the extracellular environment) and predicted secondary structure. Disulphide bond predictions were obtained using DiANNA [17], DISULFIND [52] and PreCys [18]. A secondary structure prediction from PSIPRED [53] was obtained at the Meta server [54]. These constraints were incorporated into modelling of the complete ASP-2A sequence using MODELLER.

\section{Authors' contributions}

LVM designed and performed the sequence and phylogenetic analysis and drafted the manuscript. HE performed the PCR analysis. DJR did the protein modelling study. SP conceived and planned the project. DJR and SP contributed to the analysis and interpretation of the data. All the authors read and approved the final manuscript.

\section{Additional material}

\section{Additional file 1}

Supplementary Table 1. Primer sequences used in study.

Click here for file

[http://www.biomedcentral.com/content/supplementary/1471-

2164-10-611-S1.DOCX]

\section{Acknowledgements}

This work was funded by The Natural Environment Research Council (NERC), UK. We are grateful for the assistance of staff at the Advanced Genomics Facility and the NERC Molecular Genetics Facility at the University of Liverpool, UK, at the NERC Environmental Data Centre, Oxford, UK and at The Sanger Institute, Cambridge, UK.

\section{References}

I. Szecsi PB: The aspartic proteases. Scand J Clin Lab Invest Suppl 1992, 2 1 0:5-22.

2. Dunn BM: Structure and mechanism of the pepsin-like family of aspartic peptidases. Chem Rev 2002, 1 02(I 2):443 I-4458.

3. Williamson AL, Brindley PJ, Knox DP, Hotez PJ, Loukas A: Digestive proteases of blood-feeding nematodes. Trends Parasitol 2003, 19(9):4I7-423.

4. Williamson AL, Brindley PJ, Abbenante G, Prociv P, Berry C, Girdwood K, Pritchard DI, Fairlie DP, Hotez PJ, Dalton JP, Loukas A: Cleavage of hemoglobin by hookworm cathepsin $D$ aspartic proteases and its potential contribution to host specificity. FASEB J 2002, I6 (II): 1458-1460.

5. Bethony J, Brooker S, Albonico M, Geiger SM, Loukas A, Diemert D, Hotez PJ: Soil-transmitted helminth infections: ascariasis, trichuriasis, and hookworm. Lancet 2006, 367(952I):I52I-I532.

6. Loukas A, Bethony J, Brooker S, Hotez P: Hookworm vaccines: past, present, and future. Lancet Infect Dis 2006, 6(I I):733-74I.

7. McKerrow JH, Brindley P, Brown M, Gam AA, Staunton C, Neva FA: Strongyloides stercoralis: identification of a protease that facilitates penetration of skin by the infective larvae. Exp Parasitol 1990, 70(2):|34-|43.

8. Jolodar A, Miller DJ: Identification of a novel family of non-lysosomal aspartic proteases in nematodes. Biochim Biophys Acta 1998, 1382(I):13-16

9. Dorris M, Viney ME, Blaxter ML: Molecular phylogenetic analysis of the genus Strongyloides and related nematodes. Int J Parasitol 2002, 32(12):1507-1517.

10. Evans H, Mello LV, Fang Y, Wit E, Thompson FJ, Viney ME, Paterson $\mathrm{S}$ : Microarray analysis of gender- and parasite-specific gene transcription in Strongyloides ratti. Int J Parasitol 2008, 38(II):|329-134|.

II. Thompson FJ, Mitreva M, Barker GL, Martin J, Waterston RH, McCarter JP, Viney ME: An expressed sequence tag analysis of the life-cycle of the parasitic nematode Strongyloides ratti. Mol Biochem Parasitol 2005, I 42(1):32-46.

12. Tcherepanova I, Bhattacharyya L, Rubin CS, Freedman JH: Aspartic proteases from the nematode Caenorhabditis elegans. Structural organization and developmental and cell-specific expression of asp-I. J Biol Chem 2000, 275(34):26359-26369.

13. Syntichaki P, Xu K, Driscoll M, Tavernarakis N: Specific aspartyl and calpain proteases are required for neurodegeneration in C. elegans. Nature 2002, 419(69 10):939-944.

14. Longbottom D, Redmond DL, Russell M, Liddell S, Smith WD, Knox DP: Molecular cloning and characterisation of a putative aspartate proteinase associated with a gut membrane protein complex from adult Haemonchus contortus. Mol Biochem Parasitol I997, 88(1-2):63-72.

15. Smith WD, Skuce PJ, Newlands GF, Smith SK, Pettit D: Aspartyl proteases from the intestinal brush border of Haemonchus contortus as protective antigens for sheep. Parasite Immunol 2003, 25(II- I 2):52I-530.

16. Hill AA, Hunter CP, Tsung BT, Tucker-Kellogg G, Brown EL: Genomic Analysis of Gene Expression in C. elegans. Science 2000, 290(5492):809-8I2

17. Ferre F, Clote P: DiANNA: a web server for disulfide connectivity prediction. Nucleic Acids Res 2005:W230-2.

18. Zhao E, Liu HL, Tsai CH, Tsai HK, Chan CH, Kao CY: Cysteine separations profiles on protein sequences infer disulfide connectivity. Bioinformatics 2005, 21(8): 1415-1420.

19. McGhee JD: The C. elegans intestine. [http://www.worm book.org/chapters/www intestine/intestine.html].

20. Munn EA, Munn PD: Feeding and Digestion. In The Biology of Nematodes Edited by: Lee DL. London: Taylor and Francis; 2002.

21. Delcroix M, Sajid M, Caffrey CR, Lim KC, Dvorak J, Hsieh I, Bahgat M, Dissous C, McKerrow JH: A multienzyme network functions in 
intestinal protein digestion by a platyhelminth parasite. J Biol Chem 2006, 28I(5 I):39316-39329.

22. Robertson HM: Two large families of chemoreceptor genes in the nematodes Caenorhabditis elegans and Caenorhabditis briggsae reveal extensive gene duplication, diversification, movement, and intron loss. Genome Res 1998, 8:449-463.

23. Robertson HM: Updating the str and srj (stl) families of chemoreceptors in Caenorhabditis nematodes reveals frequent gene movement within and between chromosomes. 200I, 26:15I-159.

24. Robertson HM: The large srh family of chemoreceptor genes in Caenorhabditis nematodes reveals processes of genome evolution involving large duplications and deletions and intron gains and losses. Genome Res 2000, 10:192-203.

25. Wasmuth J, Schmid R, Hedley A, Blaxter M: On the extent and origins of genic novelty in the phylum nematoda. PLoS Negl Trop Dis 2008, 2(7): 258 .

26. Paterson S, Viney ME: Functional consequences of genetic diversity in Strongyloides ratti infections. Proc Biol Sci 2003, 270(15 19): 1023-1032.

27. Paterson S, Barber R: Experimental evolution of parasite lifehistory traits in Strongyloides ratti (Nematoda). Proc Biol Sci 2007, 274( ( 6 I 7): | 467-| 474.

28. Paterson S, Viney ME: Host immune responses are necessary for density dependence in nematode infections. Parasitology 2002, I 25(Pt 3):283-292.

29. Zhu YY, Machleder EM, Chenchik A, Li R, Siebert PD: Reverse transcriptase template switching: a SMART approach for fulllength cDNA library construction. Bio Techniques 200I, 30(4):892-897.

30. Zhulidov PA, Bogdanova EA, Shcheglov AS, Vagner LL, Khaspekov GL, Kozhemyako VB, Matz MV, Meleshkevitch E, Moroz LL, Lukyanov SA, Shagin DA: Simple cDNA normalization using kamchatka crab duplex-specific nuclease. Nucleic Acids Res 2004, 32(3):e37.

31. Margulies M, Egholm M, Altman WE, Attiya S, Bader JS, Bemben LA, Berka J, Braverman MS, Chen YJ, Chen Z, Dewell SB, Du L, Fierro JM, Gomes XV, Godwin BC, He W, Helgesen S, Ho CH, Irzyk GP, Jando SC, Alenquer ML, Jarvie TP, Jirage KB, Kim JB, Knight JR, Lanza JR, Leamon JH, Lefkowitz SM, Lei M, Li J, Lohman KL, Lu H, Makhijani VB, McDade KE, McKenna MP, Myers EW, Nickerson E, Nobile JR, Plant R, Puc BP, Ronan MT, Roth GT, Sarkis GJ, Simons JF, Simpson JW, Srinivasan M, Tartaro KR, Tomasz A, Vogt KA, Volkmer GA, Wang SH, Wang Y, Weiner MP, Yu P, Begley RF, Rothberg JM: Genome sequencing in microfabricated high-density picolitre reactors. Nature 2005, 437(7057):376-380.

32. Altschul SF, Madden TL, Schaffer AA, Zhang J, Zhang Z, Miller W, Lipman D]: Gapped BLAST and PSI-BLAST: a new generation of protein database search programs. Nucleic Acids Res 1997, 25(I7):3389-3402.

33. Rogers A, Antoshechkin I, Bieri T, Blasiar D, Bastiani C, Canaran P Chan J, Chen WJ, Davis P, Fernandes J, Fiedler TJ, Han M, Harris TW, Kishore R, Lee R, McKay S, Muller HM, Nakamura C, Ozersky P, Petcherski A, Schindelman G, Schwarz EM, Spooner W, Tuli MA, Van Auken K, Wang D, Wang X, Williams G, Yook K, Durbin R, Stein LD, Spieth J, Sternberg PW: WormBase 2007. Nucleic Acids Res 2008:D6 I2-7.

34. Boutet E, Lieberherr D, Tognolli M, Schneider M, Bairoch A: UniProtKB/Swiss-Prot. Methods Mol Biol 2007, 406:89-II2.

35. Sayers EW, Barrett T, Benson DA, Bryant SH, Canese K, Chetvernin V, Church DM, DiCuccio M, Edgar R, Federhen S, Feolo M, Geer LY, Helmberg W, Kapustin Y, Landsman D, Lipman DJ, Madden TL, Maglott DR, Miller V, Mizrachi I, Ostell J, Pruitt KD, Schuler GD, Sequeira E, Sherry ST, Shumway M, Sirotkin K, Souvorov A, Starchenko G, Tatusova TA, Wagner L, Yaschenko E, Ye J: Database resources of the National Center for Biotechnology Information. Nucleic Acids Res 2009:D5-15.

36. Salamov AA, Solovyev VV: Ab initio gene finding in Drosophila genomic DNA. Genome Res 2000, 10(4):516-522.

37. Finn RD, Tate J, Mistry J, Coggill PC, Sammut SJ, Hotz HR, Ceric G Forslund K, Eddy SR, Sonnhammer EL, Bateman A: The Pfam protein families database. Nucleic Acids Res 2008:D28I-8.

38. Edgar RC: MUSCLE: a multiple sequence alignment method with reduced time and space complexity. BMC Bioinformatics 2004, 5: II3.

39. Clamp M, Cuff J, Searle SM, Barton G]: The Jalview Java alignment editor. Bioinformatics 2004, 20(3):426-427.
40. Tamura K, Dudley J, Nei M, Kumar S: MEGA4: Molecular Evolutionary Genetics Analysis (MEGA) software version 4.0. Mol Biol Evol 2007, 24(8): I596-I599.

4I. Kumar S, Nei M, Dudley J, Tamura K: MEGA: a biologist-centric software for evolutionary analysis of DNA and protein sequences. Brief Bioinform 2008, 9(4):299-306.

42. Soding J, Biegert $A$, Lupas AN: The HHpred interactive server for protein homology detection and structure prediction. Nucleic Acids Res 2005:W244-8.

43. Berman $\mathrm{H}$, Henrick $\mathrm{K}$, Nakamura $\mathrm{H}$, Markley JL: The worldwide Protein Data Bank (wwPDB): ensuring a single, uniform archive of PDB data. Nucleic Acids Res 2007:D30I-3.

44. Fujinaga M, Chernaia MM, Tarasova NI, Mosimann SC, James MN: Crystal structure of human pepsin and its complex with pepstatin. Protein Sci 1995, 4(5):960-972.

45. Dealwis CG, Frazao C, Badasso M, Cooper JB, Tickle IJ, Driessen H, Blundell TL, Murakami K, Miyazaki H, Sueiras-Diaz J: X-ray analysis at $2.0 \mathrm{~A}$ resolution of mouse submaxillary renin complexed with a decapeptide inhibitor $\mathrm{CH}-66$, based on the 4-16 fragment of rat angiotensinogen. I Mol Biol 1994, 236(I):342-360.

46. Borelli C, Ruge E, Schaller M, Monod M, Korting HC, Huber R, Maskos K: The crystal structure of the secreted aspartic proteinase 3 from Candida albicans and its complex with pepstatin A. Proteins 2007, 68(3):738-748.

47. Tong L, Pav S, Lamarre D, Pilote L, LaPlante S, Anderson PC, Jung G: High resolution crystal structures of recombinant human renin in complex with polyhydroxymonoamide inhibitors. J Mol Biol 1995, 250(2):2I I-222.

48. Krissinel E, Henrick K: Secondary-structure matching (SSM), a new tool for fast protein structure alignment in three dimensions. Acta Crystallogr D Biol Crystallogr 2004, 60(Pt 12 Pt I):2256-2268

49. DeLano WL: The PyMOL Molecular Graphics System on the World Wide Web. [http://www.pymol.org].

50. Sali A, Blundell TL: Comparative protein modelling by satisfaction of spatial restraints. J Mol Biol 1993, 234(3):779-8।5.

5I. Laskowski RA, Moss DS, Thornton JM: Main-chain bond lengths and bond angles in protein structures. I Mol Biol 1993, 23 I(4): 1049-1067.

52. Ceroni A, Passerini A, Vullo A, Frasconi P: DISULFIND: a disulfide bonding state and cysteine connectivity prediction server. Nucleic Acids Res 2006:WI77-8I.

53. Jones DT: Protein secondary structure prediction based on position-specific scoring matrices. J Mol Biol 1999 , 292(2): 195-202.

54. Bujnicki JM, Elofsson A, Fischer D, Rychlewski L: Structure prediction meta server. Bioinformatics 200I, 17(8):750-75I.

55. Baker NA, Sept D, Joseph S, Holst MJ, McCammon JA: Electrostatics of nanosystems: application to microtubules and the ribosome. Proc Natl Acad Sci USA 200I, 98( 18): 10037-I004I.

Publish with Biomed Central and every scientist can read your work free of charge

"BioMed Central will be the most significant development for disseminating the results of biomedical research in our lifetime. "

Sir Paul Nurse, Cancer Research UK

Your research papers will be:

- available free of charge to the entire biomedical community

- peer reviewed and published immediately upon acceptance

- cited in PubMed and archived on PubMed Central

- yours - you keep the copyright
BioMedcentral 